\title{
L'Émoi de l'Autre: traduire la poésie (Leopardi face à Bonnefoy, Jaccottet, Orcel)
}

\section{Fabio Scotto}

\section{(2) OpenEdition}

1 Journals

\section{Édition électronique}

URL : https://journals.openedition.org/studifrancesi/20606

DOI : 10.4000/studifrancesi.20606

ISSN : 2421-5856

Éditeur

Rosenberg \& Sellier

\section{Édition imprimée}

Date de publication : 1 décembre 2019

Pagination : 471-484

ISSN : 0039-2944

\section{Référence électronique}

Fabio Scotto, «L'Émoi de l'Autre: traduire la poésie (Leopardi face à Bonnefoy, Jaccottet, Orcel) », Studi Francesi [En ligne], 189 (LXIII | III) | 2019, mis en ligne le 01 décembre 2020, consulté le 12 novembre 2021. URL : http://journals.openedition.org/studifrancesi/20606 ; DOI : https://doi.org/10.4000/ studifrancesi.20606

\section{(c) (i) (9)}

Studi Francesi è distribuita con Licenza Creative Commons Attribuzione - Non commerciale - Non opere derivate 4.0 Internazionale. 


\title{
L'Émoi de l'Autre: traduire la poésie (Leopardi face à Bonnefoy, Jaccottet, Orcel)
}

\begin{abstract}
This article is a corrected and revised version of a paper presented at the symposium "Traduire la poésie: les significations et le sens", organised by Yves Bonnefoy and Carlo Ossola (Fondation Hugot du Collège de France, Paris, 5-6 November 2015), the proceedings of which were never published. It proposes to investigate, in the introduction and in the historical-theoretical survey, the lines of development of a school of thought in translation which has always championed meaning, in our own times overcoming defining dualities and "prejudicial objection" in favour of rhythm and form as active features of poetic subjectivity. In the third section, the analysis and comparison between three versions of Leopardi's L'infinito allows us to draw attention in Jaccottet and Orcel to a tendency towards the imitative tracing of these features, which in the latter's translation also tend towards the archaic, whilst in Bonnefoy the move away from these formal similarities corresponds to the translator's poetic imperative, relating to his notion of "traduction au sens large", and extending also to the poet-translator's own creative work.
\end{abstract}

\section{Préambule}

Le problème de la traduction poétique impose une réflexion historique préalable à même de montrer le rapport existant entre la poésie et l'origine de la littérature, ainsi que les modalités de communication du texte privilégiées par les créateurs qui permettent de comprendre les raisons pour lesquelles un certain texte a pu être traduit d'une certaine façon.

Cet aperçu historique montrera essentiellement que la théorie de la traduction dérive de sa pratique, qu'à de rares exceptions près la traduction, au moins jusqu'à la fin du XVIII siècle, a été surtout un problème de sens et que cette attitude n'est pas sans rapport avec une esthétique de la réception fondée sur l'ethnocentrisme et sur une volonté d'annexion souvent incapable de viser l'altérité de l'Autre comme valeur $^{1}$. En effet, la traduction met en jeu la relation profonde d'un sujet, mais aussi d'une culture, avec d'autres façons de penser, d'autres époques et d'autres visions du monde dont le statut est du point de vue diachronique variable, à cause de ce que Friedmar Apel appelle «le mouvement du langage» ${ }^{2}$, où tout dans la chaîne qui permet la communication d'un texte est «en mouvement», du statut de la langue aux capacités de lecture et de décodage du message, à un point tel que même l'original ne saurait être invariable, si seulement on pense à la capacité d'un lecteur moderne d'en saisir pleinement aujourd'hui, à l'aide parfois des moyens technologiques les plus

(1) A. Berman, La traduction et la lettre ou L'auberge du lointain, Paris, Seuil, 1999, «L'ordre philosophique», p. 26

(2) F. Apel, Sprachbewegung (1982), Il movimento del linguaggio, éds. E. Mattioli et R. Novello, Milano, Marcos y Marcos, 1997, «I Saggi di Testo a fronte» 8. 
avancés - comme, par exemple, la recherche numérique des occurrences lexicales et des variantes -, la stratification profonde.

Faire affleurer «le sens», dans une acception particulière non purement sémantique, signifie donc montrer avant tout la spécificité du poétique dans l'articulation du langage, mais aussi interroger la notion même de sens par rapport à la signification, qui se limiterait à la dimension purement sémantique et donc conceptuelle du discours. Or, l'on sait que ce qui fait de la poésie ce qu'elle est n'est pas seulement ce qu'elle dit, mais surtout la manière de le dire, ce comment dont dépend souvent sa capacité de susciter l'émotion, la stupeur, l'émerveillement que l'on doit au génie humain lorsqu'il se montre capable par intuition d'inventer la forme qui l'invente, de devenir par le texte ce qu'il était déjà en puissance, bref le faire de son dire.

Par-delà tout dualisme désormais périmé entre forme et sens, fidélité et infidélité, signifiant et signifié, le problème que pose la traduction aujourd'hui est essentiellement celui de la définition d'une démarche, d'une activité toujours en devenir - du fait du caractère «périssable» des traductions - qui investit la notion de rythme au sens non seulement linguistique d'articulation de la parole par des chaînes sérielles dans l'élaboration phono-sémantique du discours ${ }^{3}$, mais aussi d'identification du rapport entre l'auteur et l'auteur-traducteur et de tout ce que cette rencontre/affrontement ${ }^{4}$ peut produire chez eux et dans leurs cultures respectives.

Je me propose donc dans cet article de mettre en évidence la complexité de ce parcours d'élaboration dans sa valeur théorique et pratique, pour montrer ensuite, par une analyse comparée des versions françaises d'Yves Bonnefoy, de Philippe Jaccottet et de Michel Orcel de L'Infini de Leopardi, et par la pratique et la théorie explicite ou implicite sous-jacentes, l'étroit rapport existant entre la poétique de l'auteur et sa manière de traduire, surtout dans le cas des poètes-traducteurs. Cette démarche me permettra de montrer l'importance du son dans la définition du sens, à savoir d'une signifiance qui ne saurait pas se passer de la dimension formelle de la poésie comme comment qui dit le quoi du poème, ce qui suppose et exige de sortir de la dimension purement verbale pour atteindre la réflexion sur le sens de l'existence, donc sur son éthique qui passe nécessairement par le contact avec l'Autre et par la manière dont il nous influence et modifie. L'émoi de l'Autre (le Moi de l'Autre) sera alors le processus par lequel traduire exige que l'on se mette émotionnellement à l'écoute de l'Autre, mais aussi que l'on demande au Moi traduisant, pour ce faire, de s'investir subjectivement dans la démarche pour y laisser des traces de lui-même, soient-elles volontaires ou bien inconscientes, bref, d'aller de la transparence vers la visibilité

\section{Traduire: une petite histoire du sens. Des origines à nos jours}

D'abord, il faut rappeler que la traduction est une praxis et que sa théorie dérive de la réflexion que les traducteurs font sur leur pratique. La lecture de l'original, dont la traduction devient à bien des égards la forme la plus approfondie, est une expé-

(3) Je me réfère là à la pensée d'Henri Meschonnic qui élabore une notion de rythme non métrique définie comme «l'organisation du mouvement de la parole dans le langage» et fondée sur l'oralité du discours d'un sujet qui est créé par ce qu'il dit. Sa «poétique de la traduction» se veut une «pratique théorique» (H. Meschonnic, Pour la poétique II. Épistémologie de l'écriture. Poétique de la traduction, Paris, Gallimard, 1973, p. 305 et ss.). Voir aussi G. Dessons et H. Meschonnic, Traité du rythme. Des vers et des proses, Paris, Dunod, 1998; H. Meschonnic, Poétique du traduire, Lagrasse, Verdier, 1999.

(4) L. Anceschi, Arte, critica, filosofia, Bologna, Patron, 1965, «Studi di estetica» 1.

(5) L. Venuti, L'invisibilità del traduttore, Roma, Armando, 1999. 
rience qui se voudra, à l'origine, reproduction, pour devenir successivement œuvre d'auteur proprement dite. Les premières traductions remontent notamment au XVIII ${ }^{\mathrm{e}}$ siècle avant J.C. en Mésopotamie, quand l'épopée de Gilgamesh fut traduite en trois versions pour la rendre accessible aux Assyriens, aux Akkadiens et aux Sumériens. Ce n'est pas sans importance que les premiers textes traduits soient des textes sacrés puisqu'ensuite sera élaborée une théorie de la traduction (à partir des targumin, traductions commentées et paraphrasées) en bonne partie fruit de la réflexion sur la traduction de la Bible qui deviendra nécessaire à partir du VI ${ }^{e}$ siècle avant J.C. à cause de l'exode des Juifs à Babylone (traduction en araméen), puis en Egypte (premier projet de traduction en grec à Alexandrie d'Égypte, de la Bible des Septuaginta). Après l'hégémonie culturelle grecque, période où la traduction est somme toute peu pratiquée, c'est à Rome que la traduction des textes grecs s'intensifie et qu'on voit s'affirmer avec Cicéron une théorie normative prescriptive dont l'influence sera considérable. Dans son Libellus de optimo genere oratorum (ca. 46 avant J.C.), Cicéron affirme avoir traduit Démosthènes et Eschine «ut orator», non «ut interpres», en affirmant l'identité de l'«auctor»-traducteur qui ne traduit pas «verbum pro verbo», mais «sensum pro senso», c'est-à-dire en recréant librement l'efficacité expressive du texte. Cette méthode traductive privilégiant le sens sera adoptée par saint Jérôme dans son Liber de optimo genere interpretandi (ca. 390) et s'étendra jusqu' au XVII ${ }^{\mathrm{e}}$ siècle avec les belles infidèles ${ }^{6}$, sorte d'adaptation esthétisante à la langue d'arrivée, considérée comme supérieure à l'original. Fruit d'une mentalité «ethnocentrique» qu'Antoine Berman estime être l'un des défauts de la traduction occidentale ${ }^{7}$, cette logique de l'annexion tend à effacer toute trace de l'original et de son altérité, pour l'assimiler par dissimilation (de l'original) au système linguistique et culturel d'arrivée; de cette manière, ce qu'il appelle «[...] mauvaise traduction [...] généralement sous couvert de transmissibilité, opère une négation systématique de l'étrangeté de l'œuvre étrangère» ${ }^{8}$. Berman énumère dans l'«analytique de la traduction» les «tendances déformantes» dont se sert ce type de traduction pour réduire l'Autre au propre; elles vont de la clarification à l'allongement, de l'ennoblissement à l'homogénéisation et à la destruction des rythmes', alors que traduire se devrait d'être l'accueil dans sa propre «auberge» d'un «lointain» demeuré Autre.

Leonardo Bruni avait par contre insisté dans son De interpretatione recta (ca. 1420) sur l'importance de la connaissance des deux langues, ainsi que sur l'exigence de ne pas se borner à la connaissance de la réalité, mais aussi à la «beauté du style» et à la «parfaite conservation du rythme» de l'original, idées qu'on retrouvera un siècle après chez Étienne Dolet (De la manière de bien traduire d'une langue en l'autre, 1540). Madame Dacier et Daniel Huet ${ }^{10}$ s'étaient opposés dans leurs travaux à l'identification réductrice de la traduction au seul sens; ces grandes voix demeurent pour-

(6) L'expression vient de Gilles Ménage qui affirme, à propos des traductions de Lucien Perrot d'Ablancourt, qu'elles lui rappellent une femme qu'il a beaucoup aimée à Tours, et qui était belle mais infidèle, in La Teoria della traduzione nella storia, éd. S. Nergaard, Milano, Bompiani, 1993, p. 38. Voir aussi G. Mounin, Les belles infidèles, "Les Cahiers du Sud", Paris, 1955; Id., Teoria e storia della traduzione, Torino, Einaudi, 1965, pp. 44-51.

(7) «Ethnocentrique signifiera ici: qui ramène tout à sa propre culture, à ses normes et valeurs, et considère ce qui est situé en dehors de celle-ci - l'Étranger - comme négatif ou tout juste bon à être annexé, adapté, pour accroître la richesse de cette culture», A. Berman, La traduction et la lettre cit., p. 29.

(8) A. Berman, L'Épreuve de l'étranger. Culture et traduction dans l'Allemagne romantique, Paris, Gallimard, 1984, p. 17.

(9) A. Berman, La traduction et la lettre cit., pp. 49-68.

(10) D. Huet, De Interpretatione libri duo, quorum prior est de optimo genere interpretandi, alter de claris interpretibus (1661). 
tant assez isolées dans leur temps où l'hégémonie du sens semble s'imposer en France en tant qu'émanation culturelle de la politique du Grand Siècle.

Époque fort sensible à la traduction et à l'esprit philologique, surtout grâce à la politique culturelle de François ${ }^{\mathrm{I} r}$, fondateur des Collèges royaux, la Renaissance est aussi une période d'imitation, comme l'atteste la poétique de la Pléiade, peu confiante dans le pouvoir de la langue française d'arriver à imiter les Anciens et à les égaler, faute d'une langue capable d'«illustrer» la langue française par une grande littérature poétique ${ }^{11}$.

C'est avec le Romantisme allemand que, comme nous l'explique bien Antoine Berman, la perspective change radicalement, d'abord grâce à Goethe qui dans son Introduction à la traduction d'Agamemnon d'Eschyle (1819) parle d'une traduction identique à l'original à mettre sur le même plan que celui-ci, bref, de la sortie d'une condition ancillaire de minorité de la traduction, au profit d'une idée de traduction comme expression. Humboldt exigera une fidélité au caractère unique et original du texte-source; Schleiermacher encouragera le déplacement du lecteur vers l'auteur; Novalis définira la poésie comme traduction et Hölderlin fera de la traduction un acte poétique tellement radical qu'elle deviendra le lieu du métissage et de l'hybridation, ce qu'il appellera «la confusion des langues». L'allemand semble craquer sous ses pieds quand il traduit Sophocle en le grécisant (Pierre Klossowski donnera une sensation semblable deux siècles après en traduisant l'Énéide à travers une latinisation du français, et d'ailleurs Chateaubriand avait déjà latinisé sa traduction du Paradise lost fort latinisé de Milton). Quoi qu'on puisse dire ou penser aujourd'hui de ces traductions, ce qui compte dans le débat critique, auquel contribua beaucoup à l'époque la revue «Athenäum», c'est le fait qu'avec le Romantisme allemand la traduction commence à jouer un rôle central dans la culture, qu'on l'encourage puisque on croit qu'elle peut contribuer de manière décisive à l'enrichissement culturel et qu'on affirme la nécessité de s'ouvrir à l'Autre comme à un univers différent capable d'un rapport potentiellement fécond et maïeutique avec la culture-cible. L'on commence donc à se tourner vers l'original, à le saisir comme tel dans son altérité, au lieu de le normaliser par la belle infidélité classique visant l'assimilation et l'effacement de toute différence.

L'horizon contemporain se caractérise, d'un côté, par ce qu'on appelle «l'objection préjudicielle» de l'idéalisme de Benedetto Croce et de José Ortega y Gasset, qui semble reprendre l'argument de la rupture du «lien musaïque» dont parle Dante dans son Convivio (I, VII ${ }^{12}$. D'après Ortega y Gasset ${ }^{13}$, la traduction n'est jamais efficace parce que les synonymes ne désignent jamais exactement la même chose dans deux langues différentes et qu'on ne peut jamais reproduire la «forme interne» d'une langue dans une autre. Lui fait écho Benedetto Croce qui théorise l'impossibilité de la traduction du fait de l'impossibilité de «réduire ce qui a déjà eu sa forme esthétique à une autre forme aussi esthétique» ${ }^{14}$ et de l'inévitable différence entre la sensibilité du traducteur et celle de l'auteur qu'il traduit ${ }^{15}$.

(11) J. Du Bellay, Deffence et Illustration de la Langue Françoise, 1549.

(12) «E però sappia ciascuno che nulla cosa per legame musaico armonizzata si può de la sua loquela in altra trasmutare senza rompere tutta la sua dolcezza e armonia», ibidem.

(13) J. Ortega y Gasset, Miseria y esplendor de la traducción, 1937.

(14) B. Croce, Estetica come scienza dell' espressione e linguistica generale, Bari, Laterza, 19021 $1928^{2}, 6^{\text {ime }}$ édition revue, ch. IX, pp. 75-82, c'est moi qui traduis.

(15) Id., La Poesia. Introduzione alla critica e storia della poesia e della letteratura, Bari, Laterza, 1936, pp. 100-106, cité in La Teoria della traduzione nella storia, éd. S. Nergaard, Milano, Bompiani, 1993, p. 218. 
Ce n'est pas un hasard si Walter Benjamin cite les traductions de Sophocle par Hölderlin comme son modèle privilégié ${ }^{16}$ de traduction radicale dans son célèbre essai La tâche du traducteur (1923), où il affirme qu'«aucun poème n'est en fonction du lecteur», qu'il n'est ni communication ni message et que «la traduction est une forme ${ }^{17}$. Démarche bouleversante et singulière que Jean-René Ladmiral situe à l'origine de la position théorique «sourcière» ${ }^{18}$, celle de «[...] ceux qui, en traduction (et particulièrement, en théorie de la traduction), s'attachent au signifiant de la langue du texte-source qu'il s'agit de traduire ${ }^{19}$, position à laquelle il oppose sa position personnelle, celle des «ciblistes» qui «[...] entendent respecter le signifié (ou, plus exactement, le sens et la "valeur") d'une parole qui doit advenir dans la langue-cible» ${ }^{20}$.

Or, toute la théorie de la traduction contemporaine naît de la négation de l'«objection préjudicielle» d'origine idéaliste, comme l'explique Emilio Mattioli qui affirme l'exigence de soustraire la traduction à tout schématisme dualiste (fidèle/infidèle, littérale/libre...), car «la traduction littéraire n'est pas une équivalence de codes,

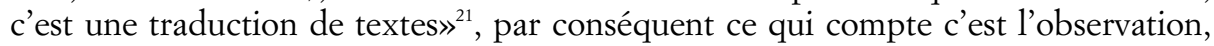
sans préjugé préalable, de toute démarche traductive par rapport à son projet initial, sa cohérence, ses résultats, qu'on doit toujours voir dans le contexte d'une histoire des traductions d'un texte, ainsi que dans le «rapport dialectique entre la poétique de l'auteur traduit et celle du traducteur» ${ }^{22}$.

La linguistique contemporaine a montré ses limites par rapport à la littérature et à la poésie dans sa prétention, qui s'est bientôt révélée illusoire, de tout expliquer grâce à la langue. Roman Jacobson ne croit pas possible la traduction de la paronomase en poésie et parle pour cela de l'exigence de la transposition créatrice ${ }^{23}$. La philosophie et l'herméneutique ont essayé avec Georg Gadamer de transposer le signifié et de faire coïncider traduire avec «comprendre» et «interpréter» ${ }^{24}$, d'après la notion formulée par Heidegger dans son Parménide qui veut que «comprendre c'est [ce soit] déjà interpréter», donc que pour comprendre il faille interpréter et que pour traduire il faille avoir compris. Le poète et linguiste Henri Meschonnic, théoricien du rythme, a reproché à Jacques Derrida «l'inclusion de la traduction dans l'interprétation ${ }^{25}$ et «l'inclusion du poème dans le sens et dans l'herméneutique», ainsi que son désir d'inclure la poésie dans la philosophie: «La poésie est au cœur de la philosophie, le poème est un philosophème» ${ }^{26}$, écrit Derrida. Pour Meschonnic la poésie est moins une question de comprendre que d'entendre, car entendre présuppose une connaissance à la fois intellective (entendre avec l'entendement) et physique, auditive (entendre des oreilles), qui implique l'investissement de la sphère orale du son dans la communication du sens, ce qu'il appelle, contre le dualisme du

(16) W. Benjamin, Die Aufgabe des Übersetzers (1923), in Gesammelte Schriften, hrsg. R. Tiedermann, H. Schweppenhäuser, Frankfurt, Suhrkamp, 1972, Bd. IV, pp. 9-21.

(17) W. Benjamin, in La Teoria cit., p. 222.

(18) J.-R. Ladmiral, "Sourciers et ciblistes", "Revue d'Esthétique" 12, nouvelle série, 1986, pp. 33-42.

(19) Ibidem.

(20) Ibidem.

(21) E. Mattioli, La traduzione letteraria, "Testo a fronte" 1, pp. 7-22, c'est moi qui traduis.

(22) Id., Studi di poetica e retorica, Modena, Mucchi, 1983, pp. 160-161.

(23) «[...] la paronomase règne dans l'art poétique. Qu'un tel domaine soit absolu ou limité, la poésie est intraduisible par définition», R. Jacobson, On Linguistic Aspects of Translation, 1959, in Teorie contemporanee della traduzione, éd. S. Nergaard, Milano, Bompiani, 1995, p. 62, c'est moi qui traduis.

(24) G. Gadamer, Wabrheit und Methode. Grundzüge einer philosophischen Hermeneutik, Tübingen,

J.C.B. Mohr, 1960, cité in Teorie contemporanee della traduzione cit., p. 345.

(25) H. Meschonnic, Poétique du traduire cit., p. 77.

(26) J. Derrida, Théologie de la traduction, in Du droit à la philosophie, Paris, Galilée, 1990, p. 337. 
signe de la sémiotique, la «forme-sens» d'un «je-ici-maintenant» ${ }^{27}$. Il s'agit, à bien des égards, d'une «poétique expérimentale»; elle historicise le poème en le situant à l'intersection du dire et du vivre et trouve son terrain d'élection dans la Bible, qui «a été et est encore le terrain premier de l'expérimentation et de la théorisation du traduire ${ }^{28}$, du fait de l'impossibilité d'y séparer, dans le verset scandé par des accents de cantillation ( $t a^{\prime} a m$, «qui signifie le goût de ce qu'on a dans la bouche» ${ }^{29}$ - pluriel: téamim) que seule la perception orale massorétique permet de saisir pleinement, le vers de la prose. Malgré la radicalité parfois excessive de certains jugements, j’estime l'apport de Meschonnic à la théorie et à la pratique du traduire considérable, non seulement par son souci de fidélité à l'original, mais surtout pour l'éthique poétique qui le caractérise, ainsi que pour l'attention à l'importance du son dans sa définition, car sans son, sans rythme et sans musique le poème n'est pas, n'a pas de force, n'arrive pas à faire ce qu'il dit. Ses idées, avec celles d'Antoine Berman, de Freidmar Apel («le mouvement du langage»), d'Efim Etkind (notamment, son idée de la «traduction-recréation $»^{30}$ ), d'Yves Bonnefoy ( $«$ la traduction au sens large $»^{31}$ ) me semblent, pour des raisons différentes, les plus importantes pour comprendre aujourd'hui dans sa complexité la spécificité de la traduction de la poésie. Pas de sens sans forme, si le mot «sens» indique aussi, si l'on en croit Octavio Paz, non seulement la valeur sémantique, mais aussi, spatialement, la direction, ce vers quoi un texte va, pourvu qu'il soit capable d'y aller.

\section{Traduire L"Infinito" de Giacomo Leopardi: Yves Bonnefoy, Philippe Jaccottet, Michel Orcel}

J'ai déjà étudié la traduction qu'a donnée Yves Bonnefoy du célèbre poème de Giacomo Leopardi L'Infini dans ma communication présentée au Colloque de Cerisy de $2005^{32}$. Si j'y reviens ici c'est surtout pour la confronter avec celles de Philippe Jaccottet et de Michel Orcel que j'ai eu le plaisir de découvrir récemment, ce qui permettra une réflexion comparée ultérieure que j'espère utile à son appréciation critique. Il faut dire d'abord que la position d'Yves Bonnefoy dans le débat critique contemporain sur la traduction est sûrement singulière; quoiqu'il semble un moment subir la tentation de l'objection préjudicielle idéaliste, du fait de l'impossibilité de faire coïncider la forme d'une langue avec celle d'une autre, il n'en fait pourtant ja-

(27) H. Meschonnic, Pour la poétique II cit., p. 286.

(28) Ibidem, p. 407.

(29) H. Meschonnic, Éthique et politique du traduire, Lagrasse, Verdier, 2007, p. 58.

(30) «Elle [la Traduction-Recréation, T-R] recrée l'ensemble, tout en conservant la structure de l'original [...] elle n'est pas possible sans sacrifices, sans transformations, sans additions», E. Etkind, Un art en crise. Essai de poétique de la traduction poétique, Lausanne, L'Age d'Homme, 1982, p. 22. Etkind affirme qu'il y a un principe dominant dans chaque poème qui n'est pas toujours le même et que le traducteur se doit de reconnaître. Une fois qu'il l'a repéré, pour être fidèle à ce principe-là (qu'il s'agisse de la rime, du mètre, de la syntaxe...) il devra renoncer à d'autres caractéristiques moins importantes de l'original pour reproduire/recréer celui-ci.

(31) Y. Bonnefoy, L'Autre langue à portée de voix. Essais sur la traduction de la poésie, Paris, Seuil, 2013, pp. $53-76$.

(32) F. Scotto, Le son de l'autre: théorie et pratique de la traduction d'Yves Bonnefoy, in Yves Bonnefoy. Poésie, recherche et savoirs, éds. D. Lançon et P. Née, Paris, Hermann éditeurs, 2007, pp. 73-92 (dont pp. 90-92, débat suivant l'intervention), puis aussi Bonnefoy e Leopardi: tra critica e traduzione, in F. Scotto, Il senso del suono. Traduzione poetica e ritmo, Roma, Donzelli, 2013, pp. 81-92, ainsi que Id., Yves Bonnefoy traducteur de Leopardi et de Pétrarque, "Littérature" 150: Yves Bonnefoy. Traduction et critique poétique, dir. P. Née, juin 2008, pp. 70-82. 
mais une raison de se rendre face à l'intraduisible et opte pour une praxis qui veut que la traduction soit toujours possible, à condition que le traducteur évite de singer l'original pour l'accueillir dans son propre monde poétique et pour le recréer librement selon l'idée que le poète-traducteur a de ce qu'il aurait voulu dire. Par conséquent, le traducteur, qui n'adhérera pas strictement à la forme de l'original, «[...] s'il écrit lui aussi il ne pourra tenir séparée sa traduction de son œuvre propre» ${ }^{33}$, s'efforcera donc de ne jamais imiter l'original, mais de le redire à sa façon, de le revivre comme expérience poétique partagée avec un Autre proche de lui-même avec lequel il a une affinité, condition cette dernière de l'expérience possible d'une rencontre. Probablement plus proche de l'attitude cibliste, au moins à première vue, à cause de sa tendance à dissimiler son texte de la forme stylistique de l'original, il se refuse pourtant au principe cibliste par excellence qui considère la traduction comme traduction essentiellement du sens («Avant tout - c'est un impératif catégorique de la pratique traduisante - il faut que le sens "passe" quoi qu'il en coûte, et fût-ce au prix d'une telle distorsion, qui fait perdre au mot le visage connu et unique qu'il présente dans l'original» ${ }^{34}$, affirme le cibliste Ladmiral), s'il écrit que «la signification n'est nullement ce qui constitue un poème [...]. La poésie ne signifie pas, elle montre. Elle ne joue pas le jeu de la signification, elle le dénie au contraire, sa raison d'être étant de se porter au-delà des représentations ${ }^{35}$. D'où le choix du vers libre et sa décision de ne point trop se soucier de reproduire les effets mimiques et phoniques de l'original, étant donné la différence de la valeur prosodique qu'ont ceux-ci lorsqu'on passe d'une langue à l'autre. Bonnefoy refuse aussi le calque, refus qui est d'après lui la condition pour la recherche d'un rapport personnel entre le rythme et le sens: «Ma traduction aussi doit être un poème: rythme et sens, produits l'un par l'autre. Mais attention: ce rythme sera le mien. Il ne pourra jamais tout à fait revivre le rythme de l'original, à cause de l'écart entre qui l'on est et qui l'on admire» ${ }^{36}$. Perplexe à l'égard de Walter Benjamin, dont il critique la notion d'Ur-Sprache, langue adamique qui lui semble être une fantaisie peu compréhensible, et en partie critique aussi envers George Steiner, pour son idée de la traduction comme transfert de signifiés ${ }^{37}$, sa position semble plus proche de celle d'Antoine Berman ${ }^{38}$, dont il fut l'ami, surtout pour la partie construens de sa démarche critique.

Bonnefoy reconnaît à Leopardi le mérite d'avoir saisi avec lucidité cinquante ans avant Mallarmé le néant du monde et d'avoir refusé, malgré son auto-identification

(33) Y. Bonnefoy, La traduction de la poésie (1976), Entretiens sur la poésie (1972-1990), Paris, Mercure de France, 1990 , p. 154.

(34) J.-R. Ladmiral, Traduire: théorèmes pour la traduction, Paris, Gallimard, 1994, «Tel», p. 220.

(35) Y. Bonnefoy, La communauté des traducteurs, Strasbourg, Presses Universitaires de Strasbourg, 2000, p. 47.

(36) Y. Bonnefoy, Traduire des Sonnets de Shakespeare, Théâtre et poésie. Shakespeare et Yeats, Paris, Mercure de France, 1998 , p. 223. Sur les traductions shakespeariennes de Bonnefoy voir les beaux ouvrages suivants: S. Amadori, Yves Bonnefoy. Père et fils de son Shakespeare, Paris, Hermann, 2015, «Savoir lettres», et M. Zach, Traduction littéraire et création poétique. Yves Bonnefoy et Paul Celan traduisent Shakespeare, Tours, Presses Universitaires François-Rabelais, 2013, «Traductions dans l'Histoire» 4. Je me permets de renvoyer également à mon article récent Yves Bonnefoy traduttore di Shakespeare. Tradurre l'opera, tradurre in opera, in Shakespeare e Cervantes (1616-2016). Traduzioni, ricezioni e rivisitazioni, a cura di F. Scotto, R. Calzoni, M. Sirtori, Cisalpino-Istituto Editoriale Universitario, Milano, 2017, «Saggi CISAM» 3, pp. 243-263.

(37) Y. Bonnefoy, Signification et poésie. Entretien avec Pierre Emmanuel Dauzat, in L'Autre langue à portée de voix cit., pp. 89-116. Pourtant George Steiner sait se libérer de «l'asservissement de la signification» et se montre sensible aux aspects «acoustiques» et à «l'incompréhensible» du texte, G. Steiner, Après Babel: une poétique du dire et de la traduction, trad. L. Lotringer et P.-E. Dauzat, Paris, Albin Michel, 1998, p. $637,555,317$.

(38) Ibidem, p. 103. 
symbolique avec les éléments naturels, de «mettre son être et la nature en état de correspondance» ${ }^{39}$. Or, il voit dans l'Ennui léopardien en tant que réponse au mal de vivre une tentation du non-être qui serait la nostalgie d'une époque théologique, celle d'un absolu personnel, à laquelle il oppose la nécessité d'accepter le monde tel qu'il est dans sa finitude en s'ouvrant au mystère de la présence qui exige que l'on soit une réalité naturelle parmi d'autres, des êtres capables d'aimer, donc d'espérer, contre toute tentation nihiliste. Sa traduction est le résultat et la conséquence de cette réflexion et elle se veut un véritable dialogue entre sa vision du monde et de la poésie et celle du poète de Recanati:

\section{L'Infinito}

Sempre caro mi fu quest'ermo colle,

E questa siepe, che da tanta parte

Dell'ultimo orizzonte il guardo esclude.

Ma sedendo e mirando, interminati

Spazi di là da quella, e sovrumani

Silenzi, e profondissima quiete

Io nel pensier mi fingo; ove per poco

Il cor non si spaura. E come il vento

Odo stormir tra queste piante, io quello

Infinito silenzio a questa voce

Vo comparando: e mi sovvien l'eterno,

E le morte stagioni, e la presente

E viva, e il suon di lei. Così tra questa

Immensità s'annega il pensier mio:

$\mathrm{E}$ il naufragar m'è dolce in questo mare ${ }^{40}$.

\section{L'Infini}

Toujours chère me fut cette colline

Solitaire; et chère cette haie

Qui refuse au regard tant de l'ultime

Horizon de ce monde. Mais je m'assieds,

Je laisse aller mes yeux, je façonne, en esprit,

Des espaces sans fin au-delà d'elle,

Des silences aussi, comme l'humain en nous

N'en connait pas, et c'est une quiétude

On ne peut plus profonde: un de ces instants

Où peu s'en faut que le cœur ne s'effraie.

Et comme alors j'entends

Le vent bruire dans ces feuillages, je compare

Ce silence infini à cette voix,

Et me revient l'éternel en mémoire

Et les saisons défuntes, et celle-ci

Qui est vivante, en sa rumeur. Immensité

En laquelle s'abîme ma pensée,

Naufrage, mais qui m'est doux dans cette $\mathrm{mer}^{41}$.

(39) Y. Bonnefoy, L'enseignement et l'exemple de Leopardi, Bordeaux, William Blake \& Co. Edit., 2001, p. 14.

(40) G. Leopardi, Canti, XII, 1819.

(41) Y. Bonnefoy, Keats et Leopardi. Quelques traductions nouvelles, Paris, Mercure de France, 2000, pp. $42-43$. 
Ce qui caractérise la traduction d'Yves Bonnefoy c'est une profonde autonomie expressive dont témoigne la liberté de la récréation structurale, où un texte monostrophique de quinze vers isosyllabiques devient un texte bi-strophique de dix-huit vers libres hétérosyllabiques. Il reproduit la déixis locative des adjectifs démonstratifs («quest'ermo colle», «queste piante, «questa voce», «questa immensità», «questo mare») avec les démonstratifs français équivalents, mais en évitant le recours aux suffixes «-ci» et «-là», un peu lourds, qui pourtant auraient donné la mesure de la différence entre la proximité et l'éloignement de la position de celui qui regarde vers l'horizon; il déplace des parties des vers de l'original d'un vers à l'autre de sa traduction (par exemple, «ermo», v. 1, «solitaire», v. 2) et supprime partiellement des hyperbates de l'original pour reconstruire l'ordre syntaxique progressif du français (vers 3, 9-11), mais tout en gardant ceux des vers 1,11 et 14 . À l'incrémentialisation ${ }^{42}$ quantitative du texte contribue sûrement une tendance explicite à la paraphrase («je laisse aller mes yeux» pour «seguendo»; «comme l'humain en nous/N'en connaît pas» pour «sovrumani», «un de ces instants» pour «ove»), ou bien à l'ajout («de ce monde», v. 4, «alors», v. 11), qui dilatent l'horizon auquel le texte fait allusion. La construction anaphorique de l'original est préservée (la conjonction «e» des vv. 8, 12-13, rendue par «et», mais par «aussi» au v. 7, pour traduire «e sovrumani»). De même, les choix lexicaux sont élégants et proches du ton et du registre de l'original. Toutefois, Bonnefoy interprète le «come» italien au vers 8 (qui signifie dès que/quand) par la modulation «comme alors» au v. 11, qui semble marquer l'écart temporel entre cette beure-ci et cette beure-là, auquel correspond, sur le plan spatial, la différence entre l'«au-delà» (v. 6, «di là da quella», v. 5) de la haie et son en deçà. Ici le français subit l'écart dû à l'impossibilité de rendre par les adjectifs déictiques démonstratifs la différence spatiale (et temporelle) entre «quest'ermo colle» (plus proche du poète, en deçà de la haie) et «quello/ infinito silenzio» (plus loin de lui, au-delà de la haie), avec toute la dialectique qui en découle, vu que ce poème commence par «quest'ermo colle» et se termine par «questo mare», pourtant entre-temps devenu l'«immensité» dans laquelle les frontières s'effacent, comme, peut-être, la haie aussi. En ce qui concerne les phonismes de l'italien, l'on constate des pertes, en particulier en ce qui concerne les nombreuses occurrences de la sibilante «s» e de l'explosive «p», aussi bien que le chiasme phonologique qui semblerait mimer l'écho de la voix:

\section{IL pEnsier mio: / E IL naufragar (vv. 14-15),}

mais on a déjà dit que Bonnefoy ne privilégie pas les imitations des effets prosodiques entre deux langues différentes.

Fondamentale à mes yeux la traduction des trois vers conclusifs, qui révèle l'homologie entre la poétique du traducteur et sa vision critique de l'œuvre de Leopardi. La suppression de «così», qui désigne la modalité (au sens de de cette manière) de l'événement, le remplacement des deux points de l'avant-dernier vers («il pensier mio:») par la virgule («ma pensée,») transformant la prolepse en apposition nominale («Naufrage,») et, enfin, la substitution de la conjonction en position d'attaque «E» («E il naufragar», dernière occurrence d'une série rythmique traçant un continu en crescendo de dix autres identiques dans l'original) par l'adversative «mais» du dernier vers («Naufrage, mais»), témoigne d'un «vouloir-être» qui verrait dans le naufrage un abandon momentané, non pas définitif, bref un instant dont la douceur («mais qui

(42) «[...] ajouts-cible au plan du signifiant et/ou au plan du signifié», J.-R. Ladmiral, Traduire: théorèmes pour la traduction cit., p. 219. 
m'est doux») atténuerait l'aspect définitif et désespéré. C'est que Bonnefoy, poète de la finitude et de l'espoir («Il n'y a rien sous les mots, sauf l'espérance», a-t-il écrit), ne peut pas considérer Leopardi comme un poète nihiliste, le nihilisme étant pour lui poétiquement inadmissible, pour ainsi dire. Ce qui ne signifie pas qu'il nie le travail du négatif mis en acte par Leopardi, travail que Bonnefoy estime utile et nécessaire, mais qu'en poésie l'amour doit toujours l'emporter sur le désarroi. De cette manière, il transforme, par un acte traductif qui se veut aussi acte critique, ce qu'on a l'habitude de connaître comme le «désespoir» leopardien en dés-espoir (à savoir l'action de l'espoir contre le désespoir à l'intérieur du désespoir lui-même), alors que la plupart des critiques (de Francesco De Sanctis à Piero Bigongiari, de Mario Luzi à Emanuele Severino ${ }^{43}$ ) ont toujours vu dans la vision du monde du grand poète italien l'impossibilité du salut et l'inévitable résignation au triomphe du néant.

Voilà maintenant la traduction du même poème par Philippe Jaccottet:

Toujours j'aimai cette hauteur déserte

Et cette haie qui du plus lointain horizon

Cache au regard une telle étendue.

Mais demeurant et contemplant j'invente

Des espaces interminables au-delà, de surhumains

Silences et une si profonde

Tranquillité que pour un peu se troublerait

Le cœur. Et percevant

Le vent qui passe dans ces feuilles - ce silence

Infini, je le vais comparant

À cette voix, et me souviens de l'éternel,

Des saisons qui sont mortes et de celle

Qui vit encor, de sa rumeur. Ainsi

Dans tant d'immensité ma pensée sombre,

Et m'abîmer m'est doux en cette mer ${ }^{44}$.

Philippe Jaccottet, qui a énormément traduit, surtout de l'allemand, du grec et de l'italien, a une approche de la traduction qui correspond à sa poétique de l'effacement, à savoir un désir du Moi de disparaître, de ne plus s'exprimer, mais tout simplement de «faire exister» ${ }^{45}$, de contempler le réel, pour l'énoncer. Sa position, si l'on en croit Mathilde Vischer, qui a consacré à son travail un ouvrage fort utile à notre analyse $^{46}$, semble à son origine a-théorique ${ }^{47}$, dans la mesure où souvent Jaccottet a traduit pour des raisons alimentaires, donc sans pouvoir toujours choisir librement

(43) F. Scotto, Il senso del suono. Traduzione poetica e ritmo cit., p. 91.

(44) Ph. Jaccottet, D'une lyre à cinq cordes (Pétrarque, Le Tasse, Leopardi, Ungaretti, Montale, Bertolucci, Luzi, Bigongiari, Erba, Góngora, Goethe, Hölderlin, Meyer, Rilke, Lavant, Burkart, Mandelstam, Skàcel), trad. Ph. Jaccottet 1946-1995, Paris, Gallimard, 1997, p. 31. Les traductions par Philippe Jaccottet de Leopardi figurent dans Leopardi, Canti, avec un choix des Euvres morales, trad. F.-A. Aulard, J. Bertrand, Ph. Jaccottet et G. Nicole, Paris, Gallimard, 1982, «Poésie», 1 ére édition: Paris, Del Duca, 1964, «Collection UNESCO». Jaccottet traduit Leopardi à la demande de Roger Caillois et il avoue, par ailleurs, sa difficulté à le traduire (M. Vischer, Philippe Jaccottet traducteur et poète. Une esthétique de l'effacement, Lausanne, 2003, «CTL (Centre de traduction littéraire de Lausanne)» 42, pp. 45, 52-53).

(45) M. Cavadini, Il poeta ammutolito. Letteratura senza io: un aspetto della postmodernità poetica. Philippe Jaccottet e Fabio Pusterla, Milan, Marcos y Marcos, 2004, «I Saggi di Testo a fronte» 13, pp. 25-26.

(46) M. Vischer, Philippe Jaccottet traducteur et poète cit.

(47) «Cet aveu, dont je crains, de surcroît, qu'il ne paraisse plus orgueilleux que modeste, c'est que je suis dans une ignorance totale des théories de la traduction (comme de celles, qui pis est, de la littérature); plus grave encore, que je n'ai jamais réfléchi aux problèmes qu'elle pose; que j’ai donc toujours pratiqué cet art de façon à peu près uniquement instinctive, pour ne pas dire à la légère», cité par M. Vischer, Philippe Jaccottet traducteur et poète cit., p. 11. 
que traduire. Cependant, cette ignorance apparente de la théorie de la traduction (d'ailleurs commune à de nombreux poètes contemporains, comme Vittorio Sereni, traducteur de Corneille, de Char, d'Apollinaire en Italie, pour ne donner qu'un exemple), n'empêche pas une éthique du traduire que Jaccottet décrit dans sa Préface d'Une lyre à cinq cordes comme l'exigence de «[...] non pas refuser l'ornement mais en éviter l'abus, abandonner tout vêtement royal ou sacerdotal pour une vêture de tous les jours», bref, de prendre acte du fait que «[...] dans le travail de la traduction, où le passage de la langue originale à l'autre, dégradant par nature puisque l'élan originel en est inévitablement absent, [Cette volonté d'《effacement»] peut requérir dans certains cas du traducteur une intervention plus libre, plus hardie, ou une réaccentuation nouvelle du chant premier ${ }^{48}$. Mais, d'après Mathilde Vischer, Jaccottet, malgré cet exercice somme toute intuitif du traduire qui «révèle un grand respect de la langue, du texte et de l'auteur dont il se fait l'intermédiaire ${ }^{49}$, vise essentiellement le ton et la voix de chaque auteur, préfère l'écoute de la singularité à la fidélité absolue et «cette voix médiane» ${ }^{50}$ proche de la notion de «transparence» dont parle Walter Benjamin dans son célèbre essai La tâche du traducteur ${ }^{51}$. C'est d'ailleurs Leopardi lui-même qui a montré l'ambition et les limites de la traduction:

Voici en quoi consiste la perfection de la traduction: l'auteur traduit ne doit pas être, par exemple, grec en italien, grec en français ou en allemand, mais tel en italien ou en allemand qu'il l'est en grec ou en français. Mais ceci est difficile et il n'est pas possible de le faire dans toutes les langues ${ }^{52}$.

Cette quête d'une équivalence horizontale du traduire valable partout dont rêve Leopardi se heurte à la différence des langues et porte sur le comment («tel [...] qu'il l'est en...») et sur l'effet, plutôt que sur le quoi.

Voyons maintenant comment Jaccottet a traduit L'Infini de Leopardi.

Comme Bonnefoy, Jaccottet a recours au vers libre et change de sujet au vers liminaire, en effet en italien c'est «l'ermo colle» qui est cher à celui qui le regarde, alors qu'ici c'est le sujet qui l'aime («Toujours j'aimai cette hauteur déserte»). Il reproduit les hyperbates aux vv. 2-3 («du plus lointain horizon/ Cache au regard») et, en général, il recrée assez fidèlement la scansion syntaxique de chaque vers, sans paraphraser ni déplacer des parties d'un vers à l'autre. La traduction de «Io nel pensier mi fingo» par «j'invente» (v. 4) semble être une hypotraduction simplifiante qui ne saisit pas la répétition spéculaire du verbe réfléchi italien («Io...mi...») - le «je façonne, en esprit» de Bonnefoy est plus proche du sens de l'original, car il sait donner l'idée de l'artifice

(48) Ph. Jaccottet, D’une lyre à cinq cordes cit., pp. 10-15. Jean-Marc Sourdillon reconnaît dans la poésie de Jaccottet «l'intention signifiante» qui la traverse et une «conception de l'acte poétique [qui] conduit peut-être à ne pas attacher trop d'importance à l'exactitude du sens des mots même si c'est par eux que passe le courant de l'émotion poétique. Les mots doivent être fidèles à une émotion et s'organiser selon la loi de l'exigence de la justesse, pour s'effacer ensuite au profit de ce qu'ils visent ou désignent (objet réel ou imaginaire, vue ou vision). Traduire c'est rejoindre cette intention de signification dans sa visée, à l'origine de son mouvement [...]», Un lien radieux. Essai sur Philippe Jaccottet et les poètes qu'il a traduits, Paris-Budapest-Turin, L'Harmattan, 2004, p. 247.

(49) M. Vischer, Philippe Jaccottet traducteur et poète cit., p.11.

(50) Ibidem, p. 17.

(51) «La transparence dont parle Benjamin rejoint la volonté d'effacement de Jaccottet, qui vise avant tout à ne pas voiler, ni cacher, ni obscurcir, ou au contraire rendre trop "lumineux" l'original. Ce pur langage $[. .$.$] est également ce que le poète cherche à atteindre», ibidem. Pourtant il me semble que la «Pure$ langue» dont parle Benjamin ne coïncide pas uniquement avec la pureté de l'expression, étant le rêve philosophique d'un retour à la phase précédant l'écriture.

(52) G. Leopardi, Tout est rien. Anthologie du Zibaldone di pensieri, trad. E. Cantavenera et B. Schefer, Paris, Allia, 1998, p. 133. 
mis en jeu par le moi avec lui-même -, alors que la reproduction des gérondifs italiens au vers 4 «Mais demeurant et contemplant», ainsi que «comparant» (v. 10) et surtout «Et percevant», qui produit une sorte de rime batelée involontaire avec «Le vent» du vers suivant, montre une appréciable attention rythmique à la reproduction des sonorités. Pour ce qui est des déictiques spatiaux, comme chez Bonnefoy ils sont perdus, tandis que la succession anaphorique des conjonctions «e» de l'original est seulement en partie reproduite et disparait aux vers 12-13 («Des saisons», «Qui vit encor»), mais elle reproduit fidèlement le «Et» de l'original italien dans la clausule finale. Mathilde Vischer fait remarquer que le verbe se souvenir utilisé par Jaccottet, qui exige la préposition de, détermine une modulation du sens, car dans l'expression me souviens de c'est le poète qui est le sujet et semble appeler à lui les souvenirs, alors que dans le mouvement original, ce sont les souvenirs que le paysage suggère qui viennent à lui ${ }^{53}$. En outre, Philippe Jaccottet semble devoir à sa pratique de la traduction de l'allemand une certaine tendance à la «tournure germanique, la substantivation $»^{54}$ et ne se refuse pas à l'imitation et au calque: «La poésie, c'est pour moi, d'abord et presque toujours une voix et un ton. Quand je traduis, j'ai l'illusion que j'entends la voix de l'écrivain, et j'essaie, très intuitivement, de l'épouser de mon mieux. De copier, de calquer» ${ }^{55}$.

Voyons maintenant la traduction de Michel Orcel poète, essayiste, psychanalyste et traducteur, entre autres, de Dante, du Tasse, de l'Arioste, d'Ippolito Nievo et de Leopardi ${ }^{56}$.

Toujours tendre me fut ce solitaire mont, et cette haie qui, de tout bord ou presque, ferme aux yeux le lointain horizon.

Mais couché là et regardant, des espaces sans limites au-delà d'elle, de surhumains silences, un calme on ne peut plus profond je forme en mon esprit, où peut s'en faut que le cœur ne défaille. Et comme j'ouis le vent bruire parmi les feuilles, cet infini silence-là et cette voix, je les compare: et l'éternel, il me souvient, et les mortes saisons, et la présente et vive, et son chant. Ainsi, par cette immensité ma pensée s'engloutit: et dans ces eaux il m'est doux de sombrer ${ }^{57}$.

Cette version du texte léopardien est probablement la plus proche de la lettre de l'original, même si elle ne semble pas la plus réussie. Elle se caractérise par la volonté de maintenir la progression syntagmatique de l'original et de traduire chaque vers sans déplacements. De même, la progression anaphorique en crescendo des conjonctions «et» est correctement reproduite. Pourtant certains calques syntaxiques

(53) M. Vischer, Philippe Jaccottet traducteur et poète cit., pp. 51-52.

(54) Ibidem, pp. 54-55.

(55) Ph. Jaccottet, Entretien avec Marion Graf, "Samedi littéraire du Journal de Genève", 18 janvier 1997, p. 30.

(56) Parmi ses traductions, je rappelle au moins celles du Roland furieux de l'Arioste (Paris, Le Seuil, 2000), de la Jérusalem libérée du Tasse (Paris, Gallimard Folio, 2002), des Chants/Canti de Leopardi (Paris, Flammarion, 2005, rééd.: Paris, GF, 2005).

(57) Ibidem, p. 49. 
de l'italien rendent la construction quelque peu lourde, qui s'efforce néanmoins de rendre visible la distance entre ce qu'il y a au-delà de la haie et ce qui est en-deçà, notamment par le suffixe «-là» au vers 10. Les choix lexicaux trahissent parfois une tendance archaïsante («le lointain horizon»); alors que l'on apprécie la recréation de la sérialité phono-prosodique du groupe vocalique «Et comme j'oü̈ le vent /bruire parm $i$ les feuilles» (v. 7-8).

Une comparaison entre les trois traductions peut montrer, au v. 13, par exemple, que «il suon di lei» est traduit respectivement par Bonnefoy et par Jaccottet par le substantif «rumeur», alors qu'Orcel le traduit plus lyriquement par «son chant».

Une comparaison ultérieure des trois traductions de la clausule peut permettre une réflexion ultérieure concernant la vision du monde du poète-traducteur par rapport à l'original.

Giacomo Leopardi

Così tra questa/

Immensità s'annega il pensier mio:/

E il naufragar m'è dolce in questo mare

Yves Bonnefoy

Immensité/

En laquelle s'abîme ma

pensée,

Naufrage, mais qui m'est doux dans cette mer
Philippe Jaccottet

Ainsi/

Dans tant d'immensité ma pensée sombre,/

Et m'abîmer m'est doux en cette mer

\author{
Michel Orcel \\ Ainsi, par cette/ \\ immensité ma pensée \\ s'engloutit:/ \\ et dans ces eaux il m'est \\ doux de sombrer
}

Chez Bonnefoy «s'abîme»-«Naufrage»-«mer» forment un continu isotopique, alors que chez Jaccottet et Orcel l'idée d'une chute vers le bas est rendue plus évidente par les verbes sombrer et s'abîmer, et surtout s'engloutir, qui témoignent d'une vision plus tragique et moins remédiable de la situation décrite qui est, chez Michel Orcel, rendue par le «par» du vers 12 plus prolongée et progressive, comme si la pensée traversait l'immensité. L'atténuation adversative n'est présente que chez Bonnefoy qui a pourtant écrit le texte le plus poétiquement réussi dans son intention d'auteur visible de par son style bien reconnaissable; mais le souci d'invisibilité $e^{58}$ par la transparence de Jaccottet se heurte à ce que Jean Starobinski appelle «le continu du chant ${ }^{59}$ et Jean-Claude Mathieu «la dimension éthique intérieure au lyrisme, le devoir du chant» ${ }^{60}$. C'est que la position traductive d'Yves Bonnefoy, qu'il précise dans son important essai «La traduction au sens large. À propos d'Edgar Poe et de ses traducteurs» ${ }^{61}$, où il propose une idée de la traduction comme «ensemble des réflexions et des décisions qui auront préparé ce texte [sa traduction] avec, tôt après

(58) «Bien que s'effacer, pour Jaccottet, ne veuille pas dire être absolument littéral, on peut considérer que, si le rythme, le ton, le sens, bref, l'inflexion première sont sensiblement modifiés par la traduction, c'est que le traducteur n'a pu «s'effacer» derrière la voix originale», M. Vischer, Philippe Jaccottet traducteur et poète cit., p. 52.

(59) J. Starobinski, Préface de Ph. Jaccottet, Poésie, Paris, Gallimard, «Poésie», pp. 8-9.

(60) J.-C. Mathieu, Philippe Jaccottet l'évidence du simple et l'éclat de l'obscur, Paris, José Corti/Les Essais, 2003, p. 547.

(61) Dans "Littérature", 150: Yves Bonnefoy. Traduction et critique poétique, dir. Patrick Née, pp. 9-24, puis in Y. Bonnefoy, L'Autre langue à portée de voix cit., pp. 53-76. 
ou en même temps, des conséquences dans sa vie et évidemment dans son œuvre propre, à divers niveaux de conscience», car «Il y a une dissémination de l'œuvre traduite dans celle du traducteur, qui aura d'ailleurs cherché le sens de ce qu'il traduit dans beaucoup plus chez l'auteur que les pages qu'il a placées sous ses yeux» ${ }^{62}$, est cohérente et à méditer, quoi qu'on puisse penser de ces prémices formelles, qui sont néanmoins celles de celui qui a écrit: «[1] a forme est une activité, elle peut être une

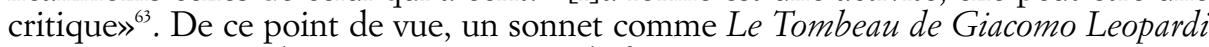
pourrait être considéré, en tant qu'acte à la fois poétique et critique, comme une sorte de «traduction au sens large», pour ce qu'il dit par «ces mots confiants» (v. 5) du «désir» de Leopardi, de «son espérance» (v. 11), de la «lune, son amie» (v. 9):

\section{Le Tombeau de Giacomo Leopardi}

Dans le nid de Phénix combien se sont

Brûlé les doigts à remuer des cendres!

Lui, c'est de consentir à tant de nuit

Qu'il dut de recueillir tant de lumière.

Et ils ont élevé, ses mots confiants,

Non le quelconque onyx vers un ciel noir

Mais la coupe formée par leurs deux paumes

Pour un peu d'eau terrestre et ton reflet,

Ô lune, son amie. Il t'offre de cette eau,

Et toi penchée sur elle, tu veux bien

Boire de ton désir, de son espérance.

Je te vois qui vas près de lui sur ces collines

Désertes, son pays. Parfois devant

Lui, et te retournant, riante: parfois son ombre ${ }^{64}$.

Position originale et profonde que celle de Bonnefoy, dense d'implications intellectuelles et opératoires, qui fait auvre personnelle de chaque poème traduit en nous disant, quelle que soit notre attitude théorique, ce que traduire fait à notre vie, à notre conscience d'êtres traducteurs, dans un vis-à-vis qui est un vie-à-vie avec le texte de l'Autre.

FABIO SCOTTO

Università degli Studi di Bergamo

(62) Y. Bonnefoy, L'Autre langue à portée de voix cit., p. 57.

(63) Y. Bonnefoy, Théâtre et poésie dans une des chroniques de Shakespeare, Préface de Shakespeare, Henri IV/Première Partie, trad. Yves Bonnefoy, Le Bruit du Temps, 2015, p. 28.

(64) Y. Bonnefoy, Le Tombeau de Giacomo Leopardi (La longue chaîne de l'ancre), in L'Heure présente et autres textes, Paris, Gallimard, 2014, «Poésie», p. 115. 